\title{
Co - Prevalence of Dengue, Chikungunya and Salmonella Infections in a Tertiary Care Hospital in South India
}

\author{
S. Nikhat Sheerin*, Mohammed Nasaruddin, V.V. Shailaja and K. Nagamani \\ Department of Microbiology, Gandhi Medical College and Hospital, Hyderabad, \\ Telangana, India \\ *Corresponding author
}

\begin{tabular}{|l|}
\hline K e y w o r d s \\
$\begin{array}{l}\text { Dengue, Chikungunya, } \\
\text { Salmonella, Typhoid, Co- } \\
\text { infection, Concurrent } \\
\text { infection, Seroprevalence }\end{array}$ \\
\hline Article Info \\
\hline $\begin{array}{l}\text { Accepted: } \\
26 \text { October } 2018 \\
\text { Available Online: } \\
10 \text { November } 2018\end{array}$ \\
\hline
\end{tabular}

A B S T R A C T

\begin{abstract}
Though dengue, chikungunya and typhoid fever have emerged as major public health problems in India, concurrent infection with these diseases is rarely reported. The present study was undertaken with the aim of determining the occurrence of dengue fever in febrile patients and co-infection with chikungunya and typhoid fever in South Indian population. Serum samples from 278 patients were tested during November- December 2017 for various parameters like Dengue IgM, Chikungunya IgM, Dengue IgG and Salmonella IgM by ELISA. The study population comprised 53\% females and $47 \%$ males with age distribution ranging from 6 months to 75 years. The serological analysis revealed a rather high prevalence of $44 \%$ acute dengue among the study population. The presence of Chikungunya infection was noted in $29 \%$ while $15 \%$ had evidence of typhoid. Interestingly, $100 \%$ of the patients tested showed dengue $\operatorname{IgG}$ seropositivity. As far as concurrent infection is concerned, $16 \%$ of the study population was found to be suffering from both dengue and chikungunya while chikungunya - Salmonella and dengue Salmonella co-infection was revealed in $7 \%$ and $5 \%$ respectively. The present study indicates the overwhelming burden of dengue, including subclinical infections, in the community as well as the significant load of concurrent infections.
\end{abstract}

\section{Introduction}

Infectious diseases are the most important causes of morbidity and mortality in developing countries, such as India. Sometimes concurrent infections with multiple infectious agents may occur, which make the correct diagnosis and management a challenging task (Uneke, 2008). Chikungunya virus (CHIKV) and dengue virus (DENV) are arboviruses currently circulating in Southeast Asia, Central and West Africa, the Pacific islands, and the Americas. Both are spread by common mosquito vectors Aedes sp. Their transmission can occur simultaneously and coinfection rates ranging from $2 \%$ to $34 \%$ have been reported (Furuya-Kanamori et al., 2016; Caron et al., 2015; Baba et al., 2013). Enteric fever or typhoid caused by Salmonella typhi is a feco-orally transmitted systemic bacterial infection that has long been endemic in our country.

Clinical features of commonly observed diseases such as malaria, enteric fever, dengue, chikungunya, scrub typhus, and 
leptospirosis mimic each other and accurately diagnosing these diseases is challenging for clinicians. Human infection with either DENV or CHIKV is associated with fever, arthralgia, malaise, headache, and rash. Typhoid fever may often present with similar clinical features. If not treated in a timely manner, coinfections may lead to life-threatening consequences (Jagadishkumar et al., 2016). In case of dengue and chikungunya, no effective vaccine or drug is available and clinicians rely on supportive therapy (Weaver et al., 2015; Dondorp et al., 2017). Specific antimicrobial therapy is available in case of typhoid which can be instituted on confirmation of diagnosis. Thus, simultaneous infections with more than one infectious agent not only complicate the diagnosis but also the course of treatment available. This study was undertaken with the aim of determining the prevalence of dengue and co-infection with chikungunya and typhoid fever in patients attending a tertiary care hospital in South India. Compilation of such data would help increase awareness among clinicians and public health authorities about the co- prevalence of these infections and help in earlier diagnosis and proper treatment of patients leading to improved outcomes.

\section{Materials and Methods}

Serum samples from 278 patients were tested in the Serology section of the Clinical Microbiology laboratory of Gandhi Hospital, attached to Gandhi Medical College, Hyderabad, Telangana during the period November-December, 2017 for the presence of Dengue $\operatorname{IgM}$ and Chikungunya $\operatorname{IgM}$ by ELISA(supplied by NIV, Pune). Of these, 103 specimens were tested for Salmonella IgM (ELISA, Calbiotech). In addition, 90 sera, out of the 278 specimens comprising the total study material, were tested for Dengue IgG (ELISA, Novalisa). All the test procedures were done as per manufacturer's instructions.
Optical density (OD) values were noted, cutoffs were calculated and results were documented. The demographic data of the patients included in the study population, namely gender and age, was also documented.

\section{Results and Discussion}

The total study population of 278 patients comprised $53 \%$ females and $47 \%$ males with a wide ranging age distribution from 6 months to 75 years.

The results of this study were expressed as percentage values (Table 1); Concomitant seroprevalence of the three infectious agents was also calculated (Table 2). The serological analysis revealed a rather high prevalence of $44 \%$ acute dengue infection among the study population. The presence of chikungunya infection was noted in $29 \%$ while $15 \%$ had evidence of typhoid. Interestingly, $100 \%$ of the patients tested showed dengue IgG seropositivity. As far as concurrent infection is concerned, $16 \%$ of the study population was found to be suffering from both dengue and chikungunya while chikungunya - Salmonella and dengue - Salmonella co-infection was revealed in $7 \%$ and $5 \%$ respectively. Percentage of patients having both $\operatorname{IgG}$ and IgM antibodies against dengue were noted to be $48 \%$ while $21 \%$ patients had presence of both CHIK IgM and DEN IgG. Concomitant seroprevalence of IgM antibodies against all three infectious agents was observed in $3 \%$ of the study population.

In developing countries such as India, infectious diseases are the most important causes of morbidity and mortality. Occurrence of concurrent infections with multiple infectious agents worsens matters further often causing increased severity of illness, complicating correct diagnosis and management leading to poorer patient outcomes. DENV and CHIKV co-infection 
rates ranging from $2 \%$ to $34 \%$ have been reported in literature (Furuya-Kanamori et al., 2016; Caron et al., 2015; Baba et al., 2013). An extensive systematic review has found evidence of malaria, dengue and chikungunya co-infections in 42 Countries spread across several geographical locations. Malaria/Dengue was the most prevalent coinfection followed by Dengue/Chikungunya (Salam et al., 2018). In Asia, the CHIKVaffected areas overlap with DENV-endemic areas (Myers et al., 1967, Mackenzie et al., 2001) and provide opportunities for mosquitoes to become infected with both viruses. In India, dengue virus causes epidemic and sporadic cases year-round, with a peak in frequency from August to November, during the humid season (Dar et al., 1999) A systematic review and metaanalysis of dengue fever in India estimated the burden of dengue fever based on published literature from India spanning over five decades. This study revealed that among the clinically suspected dengue fever patients, the estimated prevalence of laboratory-confirmed dengue infection was 38\%. It also indicated that most of the laboratory confirmed dengue cases in India occurred in young adults. The burden of dengue was also variable in studies conducted in different settings (Ganeshkumar et al., 2018). Our study revealed a rather high prevalence of $44 \%$ acute dengue infection among the study population; we also found a wide range of patients, age-wise, perhaps on account of being a major tertiary care facility in the region. Another interesting aspect of our study was the finding of $100 \%$ DENV IgG seropositivity. This may be taken as an indicator of the huge burden of dengue infections, both recent (illustrated by the high DENV IgM positivity) as well as remote, evidenced by the overwhelming percentage of DENV IgG seropositivity, in the community and the region.

Co-infections with DENV and CHIKV were reported in Calcutta, India, in 1967 (Myers et al., 1967). More recently, they were reported during the 2006 dengue outbreak in Delhi, India. Thus, in clinically suspected cases of dengue or chikungunya fever, it is advisable to test for both viruses, particularly in areas where both the viruses co circulate (Chahar et al., 2009).

Table.1 Seroprevalence of dengue, chikungunya and typhoid infections in the study population

\begin{tabular}{|l|c|c|}
\hline Name of the test (ELISA method) & Number of specimens tested & Percentage positivity (\%) \\
\hline Dengue IgM (DENV IgM) & 278 & 44 \\
\hline Chikungunya IgM (CHIKV IgM) & 278 & 29 \\
\hline Salmonella IgM (SAL IgM) & 103 & 15 \\
\hline Dengue IgG (DENV IgG) & 90 & 100 \\
\hline
\end{tabular}

Table.2 Co-prevalence of dengue, chikungunya and typhoid infections in the study population

\begin{tabular}{|c|c|}
\hline Concomitant seroprevalence & Percentage positivity (\%) \\
\hline DENV IgM + CHIKV IgM & 16 \\
\hline DENV IgM + SAL IgM & 5 \\
\hline CHIKV IgM + SAL IgM & 7 \\
\hline DENV IgM + DENV IgG & 48 \\
\hline CHIKV IgM + DENV IgG & 21 \\
\hline DENV IgM + CHIKV IgM + SAL IgM & 3 \\
\hline
\end{tabular}




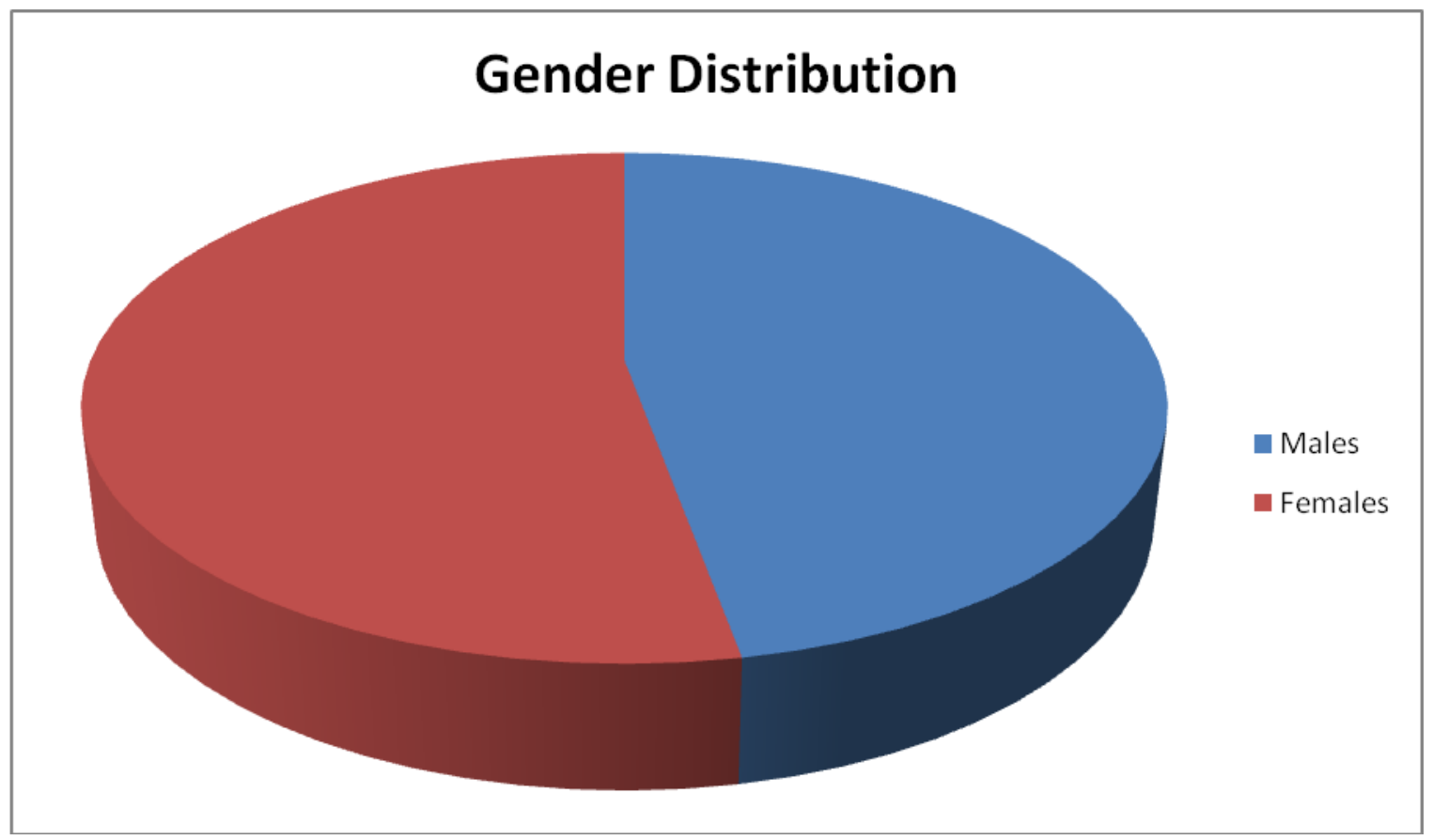

Our study findings of $16 \%$ co-prevalence of DENV-CHIKV, we believe, are on the higher side reflecting the additional burden of concomitant arboviral infections.

Enteric fever or typhoid caused by Salmonella typhi is a feco-orally transmitted systemic bacterial infection that has long been present in our country with incidence approaching one percent of the population annually in some endemic areas (Bhan et al., 2005). Coinfections with common endemic pathogens can prove to be a diagnostic challenge especially during dengue outbreaks (Srinivasaraghavan et al., 2015, Bansal et al., 2015). Risk factors for bacterial co-infection with dengue have not been well characterized. In a study done in adult patients, prolonged fever (> 5 days) was an independent risk factor for co-infection. Another probable reason cited for increased gram negative sepsis with dengue is the breakdown of intestinal mucosal barrier (Lee et al., 2005) Attempts have been made to identify risk factors predicting presence of bacterial coinfection in adult patients with dengue (See $e t$ al., 2013). A study conducted in Delhi reported dengue- typhoid co infection in $7.8 \%$ of their dengue cases (Sharma et al., 2014). The co-infection of Dengue with typhoid fever in febrile patients was 34\% according to a study conducted in Pondicherry (Kumar et al., 2017). Our study revealed a comparatively lower figure of $5 \%$ DENV- typhoid co-infection; CHIKVtyphoid co-infection also stood at a similar level of $7 \%$. Last but not the least, we found a $3 \%$ concomitant seroprevalence of IgM antibodies against all three infectious agents in our study population.

Our study had a few limitations, namely small sample size and shorter duration of study period. Also, we did not document the symptoms and related investigations of patients, which could have shed more light on the clinical features in cases of concomitant infections. All the samples tested were not processed for the presence of Salmonella IgM and this may be considered a drawback. Still, our study serves to highlight the issue of considerable numbers of patients suffering from concomitant endemic arboviral infections which deserves immediate attention 
of the public health authorities. Similar studies on a larger scale, including multicentric projects, would definitely help in elucidating the situation in its entirety.

In conclusion, co-infection should always be considered in endemic areas while dealing with patients with acute febrile illness especially when there is a prolonged fever. There is a need to document such cases and heighten awareness among clinicians regarding the changing dynamics of disease manifestations in order to provide better patient management and improve outcomes.

\section{Acknowledgement}

We would like to thank NIV, Pune, for their practical and consistent support.

\section{References}

Baba M, Logue $\mathrm{CH}$, Oderinde B, Abdulmaleek H, Williams J, Lewis J, et al., Evidence of arbovirus co-infection in suspected febrile malaria and typhoid patients in Nigeria. J Infect Dev Ctries. 2013; 7:51-9. http://dx.doi.org/10.3855/ jidc. 2411

Bansal R, Bansal P, Tomar LR. Typhoid and dengue coinfection: case reports. Trop Doct.2015; 45: 52-53.

Bhan MK, Bahl R, Bhatnagar S. Typhoid and paratyphoid fever. Lancet 366: 749762. 3; 2005

Caron M, Paupy C, Grard G, Becquart P, Mombo I, Nso BB, et al., Recent introduction and rapid dissemination of chikungunya virus and dengue virus serotype 2 associated with human and mosquito coinfections in Gabon, central Africa. Clin Infect Dis. 2012; 55: e4553.

Chahar H.S., Bharaj P., Dar L., Guleria R., Kabra S.K., and Broor S. Co-infections with Chikungunya Virus and Dengue
Virus in Delhi, India. Emerging Infectious Diseases • www.cdc.gov/eid • July 2009; Vol. 15, No. 7, 1077-1080.

Dar L, Broor S, Sengupta S, Xess I, Seth P The first major outbreak of dengue hemorrhagic fever in Delhi, India. Emerging Infect Dis; 1999.5: 589-590.

Dondorp AM, MNT H, Mer M. Recommendations for the management of severe malaria and severe dengue in resource-limited settings. In: Sepsis in Resource-Limited Settings-Expert Consensus Recommendations Group of the European Society of Intensive Care $\mathrm{M}$, the Mahidol-Oxford Research Unit in Bangkok. Intensive Care Med (2017) 43: 1683-1685 DOI 10.1007/s00134016-4602-2.

Furuya-Kanamori L, Liang S, Milinovich G, Soares Magalhaes RJ, Clements AC, Hu $\mathrm{W}$, et al., Co-distribution and coinfection of chikungunya and dengue viruses. BMC Infect Dis. 2016; 16:111. http://dx.doi.org/10.1093/cid/cis530

Ganeshkumar P, Murhekar MV, Poornima V, Saravanakumar V, Sukumaran K, Anandaselvasankar A, et al., Dengue infection in India: A systematic review and metaanalysis. PLoS Negl Trop Dis. 2018; 12(7): e0006618. https://doi.org/10.1371/journal.pntd.000 6618

Jagadishkumar K., Shareef M., Divya Hosur, Govindanahalli Shivappa Sowmya. Dengue and Typhoid Fever Coinfection in A Child. J Pediatr Inf. 2016; 10: 368.

Kumar NC, et al., Incidence of Dengue Fever in Febrile Patients and Co-Infection with Typhoid Fever in South India. Ann Med Health Sci Res. 2017; 7: 111-113

Lee I-K, Liu J-W, Yang KD () Clinical characteristics and risk factors for concurrent bacteremia in adults with dengue hemorrhagic fever. Am J Trop Med Hyg.2005; 72: 221-226 
Mackenzie JS, Chua KB, Daniels PW, Eaton BT, Field HE, Hall RA, et al., Emerging viral diseases of Southeast Asia and the Western Pacific. Emerg Infect Dis. 2001; 7: 497-504.

Myers RM, Carey DE. Concurrent isolation from patient of two arboviruses, chikungunya and dengue type 2 . Science. 1967; 157: 1307-8. DOI: 10.1126/science.157.3794.1307

Salam N., Mustafa S., Hafiz A., Chaudhary A.A., Deeba F. and Parveen S. Global prevalence and distribution of coinfection of malaria, dengue and chikungunya: a systematic review. BMC Public Health (2018) 18:710 https://doi.org/10.1186/s12889-0185626-Z

See KC, Phua J, Yip HS, Yeo LL, Lim TK. Identification of concurrent bacterial infection in adult patients with dengue. Am J Trop Med Hyg. 2013; 89: 804810.
Sharma Y, Arya V, Jain S, Kumar M, Deka L, Mathur A. Dengue and Typhoid Coinfection-Study from a Government Hospital in North Delhi. J Clin Diagn Res 2014; 8: 9-11

Srinivasaraghavan R., Narayanan P., Kanimozhi T. Culture proven Salmonella typhi co-infection in a child with Dengue fever: a case report. J Infect Dev Ctries 2015; 9(9): 10331035. doi: $10.3855 /$ jidc. 5230

Uneke CJ. Concurrent malaria and typhoid fever in the tropics: the diagnostic challenges and public health implications. J Vector Borne Dis.; 2008; 45: 133-142.

Vigna Seshan R.V., Gopalsamy S., Padma Srikanth. Dengue and typhoid coinfection: A case report from a tertiary care hospital in South India. Int J Case Rep Images 2016; 7(9): 563-565.

Weaver SC, Lecuit M. Chikungunya Virus Infections. N Engl J Med. 2015; 373: 94-5

\section{How to cite this article:}

Nikhat Sheerin, S., Mohammed Nasaruddin, V.V. Shailaja and Nagamani, K. 2018. Co Prevalence of Dengue, Chikungunya and Salmonella Infections in a Tertiary Care Hospital in South India. Int.J.Curr.Microbiol.App.Sci. 7(11): 3301-3306.

doi: https://doi.org/10.20546/ijcmas.2018.711.381 